\title{
Testing the mitigation manual on trainers
}

J C Nkomo

Energy Research Centre, University of Cape Town

\section{Introduction}

The Energy Research Centre (ERC), together with the Munasinghe Institute for Development (MIND) in Sri Lanka and the Environment and Development Action (ENDA) in Senegal, are developing training manuals to train trainers under UNITAR's Climate Change Capacity Development Programme (C3D). The rationale for this is clear. The UNFCCC seeks to strengthen non-Annex 1 countries' effective participation in the Kyoto Protocol process, and to strengthen existing and/or establish new training and research institutions to ensure sustainability of capacity building programmes. These capacity building initiatives should be country driven, involve stakeholder participation, and address specific needs and conditions of the country. For this reason, the ERC is developing training modules on climate change mitigation. ENDA is developing modules on climate change on vulnerability and adaptation; and MIND on sustainable development.

The UNITAR training is an experiment in training of trainers, which emphasises the skills element, as well as refreshing the content elements. With the focus on skills, the ERC adopted the approach that the best way to develop these without doing generic training skills courses, is to do 'learning by doing' with strong peer review. This should generate a fair degree of confidence and training skills in participants, especially if tools are well put together and supplied with detailed instructions.

Although the training manual on mitigation is prepared in South Africa, it is designed in a flexible form and can be modified for use by other countries. Consistent with $\mathrm{C} 3 \mathrm{D}$ objectives, some of expected long-term results of the training endeavour can be listed as:

- Reducing dependence on overseas institutions for skills building and capacity development;

- Increasing and sustaining abilities to develop and deliver training programme activities;

- Increasing capacity of country officials to effectively integrate into the intergovernmental climate policy debate; and;

- Integrate climate change policies into the overall national sustainable development strategy.

The ERC recently held its 'Training of Trainers'

Workshop from 5-7 April 2005. This report reviews this workshop as well assess the mitigation manual used to conduct the training. The rest of this report is divided into the following sections. The first section discusses the methodology adopted. After briefly describing the resource material for the workshop and how it works, we explain how the participants were assigned into groups to play different training roles for the duration of the workshop. The second section is on the presentation of the modules used to test the training manual. Section three presents results of the various methods used to test and get feedback on the training manual. Specifically, we want to determine the response by reviewers, what was learned, and whether the expectations of the participants were met. We attempt to solicit further feedback through evaluation forms, with results reported in the fourth section, making an overall conclusion in the last section.

\section{Objective}

The main objective of the mitigation manual is to impart skills development in the field of training, while at the same time, repeating and topping up knowledge in the content of the following mitigation courses: greenhouse gas inventories, mitigation and the clean development mechanism (CDM). Being a 'Training of Trainers Workshop', selection and invitation for attendance, apart from project partners, was based on the assumption that one is already familiar with the topics covered, or had knowledge, but did not have the skill to train or impart this knowledge. The focus was on testing whether the instructions in the training manual were clear and the training manual suitable, and to give skills to future trainers. In doing so, the goal was to familiarise the participants with a range of tasks required to conduct a 'Training of Trainers' workshop, the technical requirements necessary, and the expertise demanded.

At the start of the workshop, participants received a welcome file, which contained a welcoming letter, a $\mathrm{CD}$ on climate change mitigation, a list of participants, and the workshop agenda. The agenda allocated sufficient time for lunch and tea breaks, so that participants would make new contacts and follow up on issues raised in the sessions. Indeed there had to be a social event! Before the 
training workshop began, participants were asked to write at least one expectation they had from the course. These expectations were recorded on a flipchart page and were revisited daily as well as reviewed at the end of the workshop.

The final session gave room for participants to reflect on their learning experiences of the workshop, and to fill out evaluation forms for the entire proceedings. The importance of the evaluation is to obtain feedback on the effectiveness of the training manual, its clarity, how the training material is presented and received, and to identify areas for improvement.

\section{Methodology}

\section{Training resource material}

The training manual was designed to be used by three different groups of people: the facilitator of a course where trainers are trained; the trainers once they are trained; and the learners who are trained by the trainers. All the pages in the manual are coded at the bottom right corner of each page. Because of the highly interactive nature of the workshop, the number of participants was limited to a manageable size.

Workshop participants were given a file at the commencement of a particular course, with three training files handed out at the completion of the workshop. Each training file contained detailed instructions as well as basic material needed to conduct training, but did not include the material to be used for testing the manual or for the workshop. This material was kept in a concertina file by the Facilitator or trainer, and was handed out to participants at appropriate times. The facilitator or trainer following training instructions, is given a time indication on sections to be covered, and also follows keys and captions that help make the manuals user friendly. Although the use of concertina files for training can be disruptive, if well organised, this proves better than having pages provided in a stack up front. The material to be handed out in the concertina file was marked T, L, or G. Pages marked L (for learner) were handed out as instructed during the course. Pages marked $\mathrm{T}$ (for trainer) contain training instructions, are only handed out on completion of a particular section, and should be placed in the files at the appropriate page numbers. The handing out of the $\mathrm{G}$ (for general) pages is optional as these are, in essence, file dividers and give participants and idea of what the module is offering. The file held by a participant is complete once all these marked pages are handed out.

The training manual assumes that all adults have some knowledge on the topic, and works on the premise that adults should first explore their inherent knowledge, then apply that knowledge to 'doing' exercises. The trainer facilitates the process, and 'tops up' the learner's knowledge, thus closing off that particular learning.

\section{Training groups}

To make the workshop interesting and successful, and at the same time, maintaining its intellectual appeal, the training was organised to take the form of a rotation, so that each participant would get the opportunity to train, be trained, and observe and evaluate the trainers. As soon as the workshop preliminaries were over, and an explanation made on how to use the resource material, the course Moderator divided participants into five groups so that they would work in pairs, with participants remaining in their assigned groups for the duration of the workshop. The groups consisted of those who would attend all the sessions of the workshop. Participants not attending the full session were given the status of observer and/or evaluator. Distinct groups of participants were the Facilitator (trainer), the Learner, and the Reviewer/Evaluator. Division into these groups was done arbitrarily by the Moderator. Ten caps with five different colours were handed out arbitrarily, and once with a particular colour cap, a participant had to look for another with the same colour cap thus forming a pair for the group. The system of rotation made it possible for participants to assume different roles (to train, to be a learner and to review) with different training modules. Thus groups could be distinguished from others by the colour of their caps. The intention was for the three groups learning to always work in pairs, but as one in the context of role-plays. A necessary check was to ensure that people from the same organisation/centre were not in the same group.

Division of responsibilities to participants were as follows:

- The Moderator. The Moderator is ideally the focal person for the workshop, with responsibilities that include the following functions: organising the workshop itself, ensuring that the training materials and equipment are available to the Facilitators/ Trainers beforehand, to enable them to prepare for the workshop, and controlling group rotations and dealing with overall workshop facilitation.

- The Facilitators/Trainers. The main responsibility of the Facilitators is to facilitate the ability of the participants to train the material and their understanding of the content. Thus, Facilitators were expected to take over the training material from the previous group and commence training the next set of groups.

- The Learners. Learners had to know and understand the content so as to be able to apply it.

- The Reviewers/Evaluator. The role of the Reviewers/Evaluators was to observe the Facilitators, to make notes of their observations, 
and at the end of each rotation, to do a verbal critique of the methods, approach and evident skills of the Facilitator.

\section{Presentations}

Following the workshop agenda programme and a guide to the roles of various groups (see Appendix A), each group knew in advance the training role it would play. The design was such that each group would provide training of a module as indicated by the colour of their cap (see Appendix A), would be a reviewer for one of the modules, and for periods in between, a learner. Hence, it was vital for groups to consist of people with enough skill on the content issues to be in a position to present them fairly thoroughly. The only exception was with the exercises on Greenhouse Gas Inventories, where a specialist in the area trained the group, and also where a short-term participant was a reviewer. At the end of the training for the day, reviewers gave a report followed by a group discussion on lessons learnt.

Table 1 shows the training modules for the mitigation course, with the highlighted modules used in the workshop.

Other workshop highlights were presentations by the Munasinghe Institute for Development (MIND) on sustainable development, and ENDA on vulnerability and adaptation. These presentations came on different days, where all groups were learners and active participants in ensuring discussions. The purpose of both the MIND and ENDA presentations was to give an overview of the work being done by other project partners.

\section{Results}

\section{Reviewer's results}

Reviewers' reports to a large extent, failed to capture problems in the module. This could be because focus was largely on the delivery of the content and the style of those training, rather than on clarity of training instructions as well as the content in manuals. The comments are, however, useful hints to Facilitators and organisers in future workshops. A sample of the comments is:

- Give an overview at the beginning of each session to make it clear where we are coming from and going to

- Lack of coordination between the Facilitators in their presentations

- Encourage interaction between Facilitators and participants. There was no rapport, and with few questions to determine understanding of the presentation

- Avoid the dreadful tendency of being stationary with presentations. Rather, move around to assist participants

- Add pictures to slides to explain some of the technical concepts

- Do not talk with your back to participants. Talk to the screen and keep eye contact with participants

- Avoid reading slides to participants

- Go round and help with questions and with role plays

\section{What was learnt?}

Workshop participants expressed the following on what they learnt:

- Trainers should prepare thoroughly and go through the material beforehand

- Adult training methodology works well

- Modular approach is good, and materials can be adapted

- It is hard to write material with a step-by-step instruction

- Trainers need to target their audience

- It is important to pace the training

- Trainers should use different techniques at different times

- It is hard to use a Power Point presentation prepared by others

- Use Power Point as a 'tool' rather than as a way of presenting

\section{Were expectations met?}

Participants were asked if their expectations they had at the beginning of the course were met.

Table 1: ERC course outlines for the C3D project

\begin{tabular}{llll}
\hline Modules & & \multicolumn{2}{c}{ Courses } \\
\hline & GHG Inventories & Mitigation & CDM \\
\hline Module 1 & Introduction & Framework for mitigation & CDM and CC \\
\hline Module 2 & GHG computation & Technologies and mitigation policy & CDM and SD \\
\hline Module 3 & Exercises & Mitigation in different sectors & CDM projects \\
\hline Module 4 & GHG reporting & Cross-cutting policies & Finance and risk markets \\
\hline Module 5 & & Economic analysis & DNA \\
\hline Module 6 & & Mitigation and sustainable development \\
\hline Sections of the highlighted modules are those used for presentation in the training of trainers
\end{tabular}


Responses were:

- More than met

- Some material was at too high a level

- Learnt a lot

- All my expectations met, but there is still a lot of work to do

- The approach is innovative, even if not comfortable with the material

- I now have better understanding and confidence

- Yes, although the time was short for the three courses

- Yes, the training manual gives more effective training

- Yes, the training tool can be used effectively

\section{Workshop evaluation}

At the end of the training session before closure, the Moderator handed out evaluation forms to be filled in by all participants. The evaluation results are shown in Table 2.

\section{The challenge}

Due to financial constraints, the UNITAR delegation could not attend. There had been interest in attending, expressed by the Caribbean Community Climate Change Centre, but again budgetary constraints came in the way. These were not seen as serious impediments because our original plan was to focus on people in the Southern African region. This, however, would have required targeting spe- cialists who needed training in areas covered and allowing their organisations to cover expenses related to workshop attendance.

A major problem is that one cannot expect meaningful training three courses to be done effectively in three days. Originally, training per partner was to be for two weeks. We did not encounter language barriers with the participants from the Francophone country. A good suggestion is to try to customise our courses, so that they are not only applicable to South Africa.

The main problems pointed out in discussions for the ERC to act on are:

- GHG I should have ready exercises easy enough to follow so as to facilitate exposition;

- An example in the CDM module has inaccuracies to be attended to;

- Mitigation course could be made more interesting;

- The $\mathrm{G}$ pages should be used as dividers, or for overview or be removed; and

- Other modules should also be tested.

Overall, very positive comments were received about the training manual. The $\mathrm{ERC}$ will now incorporate the comments received during the Training of Trainers, as well as comments received in writing from ENDA, MIND and UNITAR on specific modules, in producing the final version of the training manual.

Table 2: Workshop evaluation results

\begin{tabular}{l} 
Evaluation \\
Your understanding about the \\
intention of the course? \\
\hline How well did the course achieve \\
this? (well, very well, brilliantly) \\
\hline What was your intention in \\
attending? \\
\hline Did you enjoy the course? \\
\hline Did you understand the course? \\
\hline Was the course too easy, easy, \\
and not easy enough? \\
\hline Did you struggle with the \\
language with which the course \\
was presented? \\
Which section/s did you find not \\
useful? \\
Which section/s did you find \\
useful? \\
\hline
\end{tabular}

Results/comments $(\mathbf{N}=\mathbf{1 0})$
The intention of the course was well understood by all participants,
although their responses varied as follows:
- $\quad$ To train trainers
- $\quad$ To give skills to future trainers
- To test the course material

One participant expressed that this was achieved brilliantly, while six others reported very well.

Responses to this question were largely consistent with the first one above. For three participants, the objective was to become a trainer, two wanted to get feedback on the manual and five wanted to learn more.

Six enjoyed the course very well, and two enormously.

Except for two who felt the course was not easy enough, the course was understood very easily by most (six), and easily by two.

Almost all (eight) participants found the course easy; two did not.

Only two cases reported that they struggled with the language.

Five felt that the section on GHG computation was not useful, and three pointed out that all sections were useful.

Two participants found all sections useful, three thought the CDM most useful, and another two felt the mitigation section was useful. 
Evaluation

Do you feel competent to go ahead?

What are you missing to go ahead?

What I liked about the course was ...

What I did not like about the course was ...

What lacked for me was ...

What the course could have left out was ...

Suggestions

Comment on the course methodology

Rating

Logistics and planning:

Course material

Support during the course

Office facilities

Food

Hotel and accommodation

Planning and logistics

Other logistics (please specify)

General:

Interaction with other participants

Creating a network

Becoming equipped for the tasks ahead

Were your expectations met?

What further needs do you have after the course?

Any other comments?

Author contact details

$\mathrm{J}$ C Nkomo, Energy Research Centre, University of Cape Town

Tel: +27 (0) 216502827

Fax: +27 (0) 216502830

E-mail: jabavu@erc.uct.ac.za
Results/comments $(N=10)$

Yes, from eight participants. Two felt competent but need a lot more training in other sections/modules.

There were interesting responses to this question. Six participants reported that they miss nothing to go ahead, three lack technical knowledge for some sections, and one lacks confidence.

Being called upon to play different roles was very popular (seven), the course was fun and people great (1), its interactive nature (2).

There was a $30 \%$ response to this question, with two (20\%) complaining about the long texts to read, and one about using a Power Point presentation without much preparation.

\section{No response.}

Only one responded to this, pointing out the GHG modules should have been left out given they demand time to absorb, and being of a technical nature.

Varied suggestions were received. These were: the modules should be rearranged; give more time for reading; make the $G$ pages an overview; and that the workshop should have spanned over a longer period, at least five days.

All comments received were positive, with the bulk believing the course methodology is good (six), two pointing out innovative, and one saying it was excellent.

Average out of 10

8

8

No office facilities provided.

7

Two participants in the hotel for the duration of workshop.

8

(Two responses) 7

8

7

This question received a yes from most participants (nine). Only one participant felt the technical material could have been presented in a more user-friendly manner.

Three feel the material has to be reviewed, six see no further needs, and one urges that what is left is to practise using the material

Comments ranged from OK, thanks to: the material is sufficient to improve knowledge; and that Power Point presentations need to be supplemented with audio-visual material. 
Appendix A: Roles for groups

\begin{tabular}{|c|c|c|c|c|}
\hline Day & Module & Trainer & Learner & Critique \\
\hline \multirow{2}{*}{$\begin{array}{l}\text { Day 1, } \\
\text { April 5 } \\
\text { Tuesday }\end{array}$} & GHGI & White Cap (Group 1) & $\begin{array}{l}\text { Blue Cap (Group 2) } \\
\text { Black Cap (Group 3) }\end{array}$ & Cream Cap (Group 5) \\
\hline & GHGI Exercises & GHGI specialist & Khaki Cap (Group 4) & \\
\hline \multirow{5}{*}{$\begin{array}{l}\text { Day 2, } \\
\text { April } 6 \\
\text { Wednes- } \\
\text { day }\end{array}$} & $\overline{\mathrm{CDM}} 1$ & Blue Cap (Group 2) & White Cap (Group 1) & Black Cap (Group 3) \\
\hline & & & Khaki Cap (Group 4) & \\
\hline & CDM 2 & Black Cap (Group 3) & White Cap (Group 1) & Khaki Cap (Group 4) \\
\hline & & & Blue Cap (Group 2) & \\
\hline & & & Cream Cap (Group 5) & \\
\hline \multirow{6}{*}{$\begin{array}{l}\text { Day 3, } \\
\text { April } 7 \\
\text { Thursday }\end{array}$} & Mitigation 1 & Khaki Cap (Group 4) & White Cap (Group 1) & Blue Cap (Group 2) \\
\hline & & & Black Cap (Group 3) & \\
\hline & & & Cream Cap (Group 5) & \\
\hline & Mitigation 2 & Cream Cap (Group 5) & Blue Cap (Group 2) & White Cap (Group 1) \\
\hline & & & Black Cap (Group 3) & \\
\hline & & & Khaki Cap (Group 4) & \\
\hline
\end{tabular}

